S. Takeuchi

Nagoya Math. J.

Vol. 57 (1974), 121-138

\title{
ON COMPLETENESS OF HOLOMORPHIC PRINCIPAL BUNDLES
}

\author{
SHIGERU TAKEUCHI
}

\section{§ 0. Introduction}

In this paper we shall investigate the structure of complex Lie groups from function theoretical points of view. A. Morimoto proved in [10] that every connected complex Lie group $G$ has the smallest closed normal connected complex Lie subgroup $G_{e}$, such that the factor group $G / G_{e}$ is Stein. On the other hand there hold the following two basic structure theorems $\left(A_{1}\right)$ and $\left(A_{2}\right)$ for a connected algebraic group $G$ (cf. [12]). $\left(A_{1}\right): G$ has the smallest normal algebraic subgroup $N$ such that the factor group $G / N$ is an affine algebraic group. Moreover $N$ is a connected central subgroup. $\left(A_{2}\right): G$ has the unique maximal connected affine algebraic subgroup $L$, where $L$ is normal and the factor group $G / L$ is an abelian variety.

It is well known that for algebraic groups "affine" and "linear" are equivalent, but for complex Lie groups "affine" i.e. "Stein" does not imply "linear" although the converse implication is true (cf. [9]). Nevertheless we may roughly say that Stein groups correspond to affine algebraic groups. In such a sense Morimoto's result can be considered as the analytic version of $\left(A_{1}\right)$. Considering that complex tori correspond to abelian varieties, we can formulate the analytic version of $\left(A_{2}\right)$, which is, however, not true in general. We shall prove, for reductive complex Lie groups, a structure theorem (Theorem 23) analogous to $\left(A_{2}\right)$. In general every connected complex Lie group $G$ admits a bundle structure over a complex torus, whose fibre is a Stein manifold and whose structure group is a Stein subgroup of $G$ (Corollary 25). We are mainly concerned about the dimension of the complex torus which appears in the above fibreing of $G$. We shall find that it is independent of the group structure of $G$, but depends only on the underlying complex struc-

Received March 23, 1974. 
ture of $G$ (Proposition 17, Corollary 38).

By [8] we know that every holomorphic principal bundle over a Stein manifold with a Stein fibre is Stein. In $\S 3$ we shall generalize this result to $q$-complete manifolds (Theorem 36), as follows; if $P=P(B, G)$ is a holomorphic principal bundle over a $q$-complete manifold $B$ with a connected $r$-complete structure group $G$, then $P$ is $(q+r)$-complete.

Throughout the present paper, every manifold is assumed to have countable topology. We denote by $Z, Q, R, C$, and $C^{*}$, the ring of integers, the field of rational numbers, the field of real numbers, the field of complex numbers and the group of non-zero complex numbers respectively.

I wish to express my sincere gratitude to Professor A. Morimoto and Professor K. Kasahara for their kind advices during the preparation of the present paper, and also to Doctor H. Kazama whose suggestions, given in his correspondence, motivated me to complete the latter half of of this work.

\section{§ 1. Fundamental relations of indices}

First we define four indices of complex Lie groups, and next we study their properties. Let $G$ be a connected complex Lie group with the Lie algebra $g$. Let $K$ be a maximal compact subgroup of $G$ with the Lie algebra $\mathfrak{f}$. Then $\mathfrak{f}^{C}:=\mathfrak{f}+\sqrt{-1} \mathfrak{f}$ is a complex Lie subalgebra of g. By [7] $G$ is analytically homeomorphic to $K^{C} \times C^{\alpha}$, where $K^{C}$ is a conneted complex Lie subgroup of $G$ whose Lie algebra is $\mathfrak{f}^{C}$, and $C^{\alpha}$ is a complex vector space of dimension $\alpha$. On the other hand $\mathfrak{f}_{0}:=\mathfrak{i} \cap$ $\sqrt{-1}$ is a complex Lie subalgebra of $g$, determined uniquely by $G$, independently of the choice of $K$ (cf. [10]). Since $\operatorname{dim}_{R} \mathfrak{f}^{C}=\operatorname{dim}_{R}+$ $\operatorname{dim}_{R} \sqrt{-1} \mathfrak{f}-\operatorname{dim}_{R} \cap \sqrt{-1} \mathfrak{f}$ and since $\operatorname{dim}_{R} \mathfrak{f}$ is independent of the choice of $K$, we obtain the following

Definition 1. $\alpha(G):=\operatorname{dim}_{C} G-\operatorname{dim}_{C} K^{C}$ is called the complex characteristic index of $G$.

DEFINITION 2. $\beta(G):=\operatorname{dim}_{C} \mathfrak{f}_{0}$

Definition 3. $\gamma(G):=\operatorname{dim}_{R} K$ is called the characteristic coindex of $G$.

For a complex space $X$ we denote by $\delta(X)$ the complex dimension of 
$X$. We denote by $H(X)$ the set of all holomorphic functions on $X$. For a point $x \in X$, the set $X_{x}=\left\{y \in X \mid f(x)=f(y),{ }^{\forall} f \in H(X)\right\}$ is a subvariety of $X$. It is easy to verify the following

Proposition 4. Let $\sigma$ be a complex analytic automorphism of $X$. Then we have $X_{\sigma(x)}=\sigma\left(X_{x}\right)$ for $x \in X$. Especially, if $x$ is fixed by $\sigma$, $X_{x}$ is invariant under $\sigma$.

DEFINITION 5. $\tau(X):=\inf _{x \in X} \delta\left(X_{x}\right)$ is called the holomorphic degeneracy of $X$.

Definition 6. A complex manifold $M$ is called $(H, C)$ if $H(M)=C$ (cf. [10]).

From Definitions 5, 6 we have immediately

Proposition 7. A connected complex manifold $M$ is $(H, C)$ if and only if $\tau(M)=\delta(M)$.

For a connected complex Lie group $G$, the following three conditions are equivalent (cf. [8], [10]).

(i) $G$ is Stein,

(ii) $\tau(G)=0$,

(iii) $\beta(G)=0$.

Proposition 8. For a connected complex Lie group $G$ there holds the following equality:

$$
\alpha(G)-\beta(G)+\gamma(G)-\delta(G)=0 .
$$

Proof. $\delta(G)-\alpha(G)+\beta(G)=\operatorname{dim}_{C}(\mathfrak{f}+\sqrt{-1} \mathfrak{f})+\operatorname{dim}_{C}(\mathfrak{f} \cap \sqrt{-1} \mathfrak{l})=$ $\frac{1}{2}\left(\operatorname{dim}_{R} \mathfrak{f}+\operatorname{dim}_{R} \sqrt{-1} \mathfrak{l}\right)=\operatorname{dim}_{R} \mathfrak{f}=\gamma(G) \quad$ q.e.d.

Proposition 9. For a closed normal connected complex Lie subgroup $N$ of $G$, there hold the following equalities and inequalities:

$$
\begin{gathered}
\delta(N)+\delta(G / N)=\delta(G) \\
\gamma(N)+\gamma(G / N)=\gamma(G) \\
\beta(N)+\beta(G / N) \geqq \beta(G) \geqq \beta(N) \\
\alpha(N)+\alpha(G / N) \geqq \alpha(G) \geqq \alpha(G / N) \\
\delta(G)-\tau(G) \geqq \alpha(G) .
\end{gathered}
$$


Proof. (1.2) is evident. (1.3) follows from the fact that for a maximal compact subgroup $K$ of $G, K \cap N$ and $K N / N$ are maximal compact subgroups of $N$ and $G / N$ respectively (cf. [3] p. 533 Lemma 3.15). (1.4) is proved in the following way. By the above remark $\beta(N)=$ $\operatorname{dim}_{C}(\mathfrak{f} \cap \mathfrak{n}) \cap \sqrt{-1}(\mathfrak{f} \cap \mathfrak{n})$, where $\mathfrak{n}$ denotes the Lie algebra of $N$. Since $\mathfrak{n}$ is a complex Lie algebra we have $\beta(N)=\operatorname{dim}_{C}\left(\mathfrak{f}_{0} \cap \mathfrak{n}\right) \leqq \beta(G)$. On the other hand denoting the natural surjection from $\mathrm{g}$ onto $\mathrm{g} / \mathfrak{n}$ by $\pi_{*}$ we have that $\pi_{*}(\mathfrak{f})_{0}:=\pi_{*}(\mathfrak{f}) \cap \sqrt{-1} \pi_{*}(\mathfrak{l})=\pi_{*}(\mathfrak{f}) \cap \pi_{*}(\sqrt{-1} \mathfrak{f}) \supset \pi_{*}\left(\mathfrak{f}_{0}\right)$ which concludes the former inequality of (1.4). Since $\alpha(N, G):=\alpha(N)+\alpha(G / N)$ $-(G)=\beta(N, G):=\beta(N)+\beta(G / N)-\beta(G)$ the former inequality of (1.5) follows directly from (1.4). The latter inequality of (1.5) follows from the fact that $\pi_{*}\left(\mathfrak{f}^{C}\right)=\pi_{*}(\mathfrak{f})^{C}:=\pi_{*}(\mathfrak{f})+\sqrt{-1} \pi_{*}(\mathfrak{f})$. (1.6) is an immediate consequence of the fact that $K^{C}$ contains $G_{e}$ (cf. [8], [10]).

q.e.d.

Proposition 10. Let $G$ and $\tilde{G}$ be connected complex Lie groups. If there exists a complex analytic homomorphism $\pi$ from $\tilde{G}$ onto $G$ such that the kernel of $\pi$ is a discrete subgroup of $\tilde{G}$, then there hold the following relations:

$$
\begin{aligned}
\delta(G) & =\delta(\tilde{G}) \\
\gamma(G) & \geqq \gamma(\tilde{G}) \\
\beta(G) & \geqq \beta(\tilde{G}) \\
\alpha(G) & \leqq \alpha(\tilde{G}) .
\end{aligned}
$$

Furthermore if the kernel of $\pi$ is finite, the equalities hold.

Proof. (1.2) is evident. First we prove the equalities in case the kernel of $\pi$ is finite. Let $K$ be a maximal compact subgroup of $G$. Then $\pi^{-1}(K)$ is a compact subgroup of $\tilde{G}$, hence contained in some maximal compact subgroup $\tilde{K}$ of $\tilde{G} . \pi(\tilde{K})$ is a compact subgroup of $G$ and contains a maximal compact subgroup $K$ of $G$. Hence we obtain $\pi(\tilde{K})=K$, which proves $\tilde{\mathfrak{l}} \cong \mathfrak{H}$, where $\tilde{\mathfrak{l}}$ and $\mathfrak{f}$ denote the Lie algebras of $\tilde{K}$ and $K$ respectively. Since $\pi$ induces the complex Lie algebra isomorphism $\pi_{*}$ from $\tilde{\mathfrak{g}}$ onto $\mathfrak{g}$, we get $\tilde{\mathfrak{l}}^{C} \cong \mathfrak{l} C$ and $\tilde{\mathfrak{l}}_{0}:=\tilde{\mathfrak{l}} \cap \sqrt{-1} \tilde{\mathfrak{l}} \cong \mathfrak{l} \cap \sqrt{-1} \mathfrak{l}=\mathfrak{l}_{0}$. Thus we obtain the equalities $\gamma(G)=\gamma(\tilde{G}), \beta(G)=\beta(\tilde{G})$ and $\alpha(G)=\alpha(\tilde{G})$. Secondly we prove the inequalities in case $\operatorname{ker} \pi$ is discrete. Let $\tilde{K}$ be a maximal compact subgroup of $\tilde{G}$. Then $\pi(\tilde{K})$ is a compact subgroup of $G$, hence contained in some maximal compact subgroup $K$ of $G$. 
Since $\pi_{*}$ is an isomorphism, we can consider $\tilde{\mathfrak{l}}$ as a subalgebra of $\mathfrak{f}$ by identifying $\tilde{\mathrm{g}}$ with $\mathrm{g}$. Evidently we have $\tilde{\mathfrak{f}}^{C} \subset \mathfrak{l}^{C}$ and $\tilde{\mathfrak{f}}_{0} \subset \mathfrak{f}_{0}$. Thus we obtain the inequalities (1.3)' and (1.4)'.

If a complex lie group $G$ is not assumed to be connected, we denote by $G^{0}$ the connected component of $G$ which contains the neutral element of $G$.

DEFINITION 11. $\alpha(G):=\alpha\left(G^{0}\right) . \quad \beta(G):=\beta\left(G^{0}\right) . \quad \gamma(G):=\gamma\left(G^{0}\right)$.

Proposition 12. For a complex Lie group $G$ and its closed normal complex Lie subgroup $N$ there holds the following relation:

$$
\beta(G) \leqq \beta(N)+\beta(G / N) .
$$

Proof. Since $N^{0}$ is a closed connected normal complex Lie subgroup of $G$ (and consequently of $G^{0}$ ), we can consider the factor group $G / N^{0}$ and $G^{0} / N^{0}$. Then $G^{0} / N^{0}$ is the connected component of $G / N^{0}$ since $G^{0} / N^{0}$ is connected and of the same dimension as $G / N^{0}$. Thus $G^{0} / N^{0}$ is an open and closed subset of $G / N^{0}$. Since the canonical surjection $\pi$ from $G / N^{0}$ onto $G / N$ is open and continuous, the image $\pi\left(G^{0} / N^{0}\right)$ is an open connected subgroup of $G / N$. Hence $\pi\left(G^{0} / N^{0}\right)$ is closed in $G / N$ and of the same dimension as $G / N$, which concludes that $\pi\left(G^{0} / N^{0}\right)=(G / N)^{0}$. Since $G^{0} / N^{0}$ is a covering group of $(G / N)^{0}$, we get the desired relation: $\beta\left(G^{0} / N^{0}\right) \leqq \beta(G / N)$. Thus $(1.4)^{\prime \prime}$ is proved.

For later reference we prepare the following

Proposition 13. $\gamma$ is topologically invariant i.e. if $G$ and $G^{\prime}$ are homeomorphic we have $\gamma(G)=\gamma\left(G^{\prime}\right)$.

Proof. Let $K$ and $K^{\prime}$ be maximal compact subgroups of $G^{0}$ and $G^{\prime 0}$ respectively. Let $p=\gamma(G)$ and $p^{\prime}=\gamma\left(G^{\prime}\right)$. Since $K$ and $K^{\prime}$ are orientable, $H^{p}\left(G^{0}, \mathrm{Z}\right)=H^{p}(K, \mathrm{Z})=Z$ and $H^{p^{\prime}}\left(G^{\prime 0}, Z\right)=H^{p^{\prime}}\left(K^{\prime}, Z\right)=Z$. For any $q(>p), H^{q}\left(G^{\prime 0}, Z\right)=H^{q}\left(K^{\prime}, Z\right)=0$. It follows that $p \geqq p^{\prime}$. We also have the converse inequality $p^{\prime} \leqq p$.

q.e.d.

\section{§ 2. (H.C)-Subgroups and Stein subgroups}

Let $G$ be a connected complex Lie group with the neutral element $e$.

Definition 14. A connected closed complex Lie subgroup $N$ of $G$ is called an $(H, C)$-subgroup of $G$ if $\tau(N)=\delta(N)$. 
Definition 15. A connected closed complex Lie subgroup $N$ of $G$ is called a Stein subgroup of $G$ if $\tau(N)=0$.

By [10] $G_{e}=\{x \in G \mid f(x)=f(e), \forall f \in H(G)\}$ is an $(H, C)$-subgroup of $G$. Let $N$ be an $(H, C)$-subgroup of $G$. Then we have $G_{e} \supset N$, since $G_{e} \supset G_{e} \cap N \supset N_{e}=N$. We have $\beta\left(G_{e}\right) \leqq \beta(G) \leqq \beta\left(G_{e}\right)+\beta\left(G / G_{e}\right)=\beta\left(G_{e}\right)$ by (1.4) since $G / G_{e}$ is Stein (cf. [10]). Thus we obtain

Proposition 16. $G_{e}$ is the unique maximal $(H, C)$-subgroup of $G$ and moreover for any closed normal connected complex Lie subgroup $N$ of $G$ such that $G_{e} \subset N \subset G$, there holds the equality: $\beta(G e)=\beta(N)=\beta(G)$.

Let $G$ be holomorphically homeomorphic to another complex Lie group $G^{\prime}$. Then there exists a holomorphic homeomorphism from $G$ onto $G^{\prime}$, which preserves the unit elements of the respective groups. By Proposition 4 we get $G_{e} \cong G_{e}^{\prime}$. Then $\beta\left(G_{e}\right)=\delta\left(G_{e}\right)-\gamma\left(G_{e}\right)=\delta\left(G_{e}^{\prime}\right)-\gamma\left(G_{e}^{\prime}\right)$ $=\beta\left(G_{e}^{\prime}\right)$, since $\alpha\left(G_{e}\right)=\alpha\left(G_{e}^{\prime}\right)=0$. Hence we obtain

Proposition 17 (cf. Corollary 38). $\beta$ is an analytic invariant i.e. if $G \approx G^{\prime}, \beta(G)=\beta\left(G^{\prime}\right)$.

Let $K_{0}$ be the connected complex Lie subgroup of $G$ whose Lie algebra equals $\mathfrak{l}_{0}=\mathfrak{i} \cap \sqrt{-1}$. $\quad K_{0}$ is not closed in general but is contained in $G_{e}$ (cf. [9]). We prove

Proposition 18. $G_{e}$ is the smallest closed complex Lie subgroup of $G$, which contains $K_{0}$.

Proof. Let $N$ be the smallest closed complex Lie subgroup of $G$ which contains $K_{0}$. Since $G_{e}$ contains $N$ and $G_{e}$ is central, so is $N$. Let $\mathfrak{n}$ be the Lie algebra of $N$. Then $\beta(N)=\operatorname{dim}_{C}\left(\mathfrak{f}_{0} \cap \mathfrak{n}\right)=\operatorname{dim}_{C} \mathfrak{f}_{0}=$ $\beta(G)=\beta\left(G_{e}\right)$, since $\mathfrak{n} \subset \mathfrak{f}_{0}$. On the other hand $(\mathfrak{f} \cap \mathfrak{n})+\sqrt{-1}(\mathfrak{f} \cap \mathfrak{n})$ contains $\mathfrak{f}_{0}$, and the connected complex Lie subgroup of $N$, whose Lie algebra equals $(\mathfrak{f} \cap \mathfrak{n})+\sqrt{-1}(\mathfrak{f} \cap \mathfrak{n})$, is closed in $N$ (cf. [7]). Hence we get $\mathfrak{n} \subset(\mathfrak{k} \cap \mathfrak{n})+\sqrt{-1}(\mathfrak{t} \cap \mathfrak{n})$ from the minimality condition on $N$. The converse inclusion is evident, hence $\alpha(N)=0$. For $G e$ also, we obtain $\alpha\left(G_{e}\right)=0$ by (1.6), since $\tau\left(G_{e}\right)=\delta\left(G_{e}\right) . \quad G_{e} / N$ is also $(H, C)$ hence $\alpha\left(G_{e} / N\right)=0$. By applying (1.1) (1.3) to the pair $G_{e} \triangleright N$, we obtain $\beta\left(G_{e} / N\right)=\beta\left(G_{e}\right)-\beta(N)=0$. Thus $G_{e} / N$ is at the same time Stein and $(H, C)$, which is possible only when $N=G_{e}$. q.e.d.

Now we proceed to a characterization of Stein subgroups. 
LEMma 19. Let $G$ be a connected abelian complex Lie group. Then there exists a maximal Stein subgroup $L$ such that $\delta(L)=\delta(G)-\beta(G)$. Furthermore for any maximal Stein subgroup $N$ of $G$, the factor group $G / N$ is a complex torus of dimension not smaller than $\beta(G)$.

Proof. (i) First we prove the existence of a non-trivial Stein subgroup for a non-compact group $G$. Let $\delta(G)=m, \tau(G)=n$ and $\beta(G)=r$, with $n>r$ or equivalently $m>r$. By [6] $G$ is decomposed into the direct sum of a Stein subgroup $S \cong C^{p} \oplus C^{* q}$ and the $(H, C)$-subgroup $G_{e}$. If $p+q>0$, then $S$ is a non-trivial Stein subgroup. We assume now $p+q=0$ i.e. $G=G_{e}$. Let $C^{n}$ be the universal coveing group of $G$, and let $\Lambda$ be the kernel of the covering homomorphism $\pi$ from $C^{n}$ onto $G$. $\Lambda$ is a discrete subgroup of $C^{n}$. By identifying $C^{n}$ with $\mathfrak{g}$, we can identify $\Lambda \otimes_{Z} \boldsymbol{R}$ with $\mathfrak{f}$. Thus $\gamma(G)=\operatorname{dim}_{\boldsymbol{R}} \mathfrak{f}$ equals $\operatorname{rk}_{\boldsymbol{R}} \Lambda:=\#$ maximal system of generators of $\Lambda$ which are linearly independent over $R$ \}. Since $\alpha(G)=0$ by (1.6), we get $\gamma(G)=n+r$ by (1.1). $\alpha(G)=0$ means that $\left(\Lambda \otimes_{z} \boldsymbol{R}\right)^{C}=\boldsymbol{C}^{n}$. Thus we can choose the generators $\left(e_{1}, \cdots, e_{n}, d_{1}\right.$, $\cdots, d_{r}$ ) of $\Lambda$, which are linearly independent over $R$, in such a way that $\left\{e_{1}, \cdots, e_{n}\right\}$ constitute a base of the complex vector space $C^{n}$. Thus for a suitable complex $(r, n)$-matrix $A=\left(a_{i j}\right)_{\substack{1 \leq i \leq r \\ 1 \leq j \leq n}}$, we have $d_{i}=\sum_{j=1}^{n} a_{i j} e_{j}$ $(i=1, \cdots, r)$. Let $\operatorname{Re} A:=\left(\operatorname{Re} a_{i j}\right)$, and $\operatorname{let} \operatorname{Im} A:=\left(\operatorname{Im} a_{i j}\right)$. Then $\operatorname{Re} d_{i}:=\sum_{j=1}^{n} \operatorname{Re} a_{i j} e_{j} \in \mathcal{F}$, where $\xi$ is a real vector subspace of $\mathrm{g}=C^{n}$ spanned by $\left\{e_{1}, \cdots, e_{n}\right\}$ over $R$. Evidently $\mathfrak{g}=\mathfrak{S}^{C}:=\mathfrak{g}+\sqrt{-1} \mathfrak{g}$ and $\xi \ni \operatorname{Im} d_{i}:=\sum_{j=1}^{n} \operatorname{Im} a_{i j} e_{j}$. Since $\left\{e_{1}, \cdots, e_{n}, d_{1}, \cdots, d_{r}\right\}$ are linearly independent over $R$ and since $\left\{\operatorname{Re} d_{1}, \cdots, \operatorname{Re} d_{r}\right\} \subset \xi$, thus $\sqrt{-1}\left\{\operatorname{Im} d_{1}, \cdots\right.$, $\left.\operatorname{Im} d_{r}\right\}$ are inearly independent over $R$ which implies that $\left\{\operatorname{Im} d_{1}, \cdots, \operatorname{Im} d_{r}\right\}$ span the $r$-dimensional real vector subspace $a$ of $g$. Without loss of generality, we may assume $a$ to be spanned by $\left\{e_{1}, \cdots, e_{r}\right\}$ over $\boldsymbol{R}$. Denoting by $\mathfrak{b}$ the real subspace of $\mathfrak{g}$ spanned by $\left\{e_{r+1}, \cdots, e_{n}\right\}$, we have the direct sum decomposition $\mathfrak{g}=\mathfrak{g}^{C}=\mathfrak{g} \oplus \sqrt{-1} \mathfrak{g}=\mathfrak{a}^{C} \oplus \mathfrak{b}^{C}=(\mathfrak{a} \oplus \sqrt{-1} \mathfrak{a}) \oplus$ $(\mathfrak{b} \oplus \sqrt{-1} \mathfrak{b})$. Let $\pi$ be the canonical projection from $\mathfrak{g}$ onto $\mathfrak{g} / \mathfrak{b}^{C}$. Then $\pi(\Lambda)$ is a finitely generated subgroup of the additive group $\pi(\mathfrak{g}) \cdot \mathfrak{b}^{C}+\Lambda$ is closed in $g$ if and only if $\pi(\mathcal{A})$ is closed in $\pi(\mathrm{g})$. For a finitely generated subgroup $\pi(\Lambda)$ of $\pi(\mathrm{g})$, let $\#$ \{maximal system of generators of $\pi(\Lambda)$ which are linearly independent over $\boldsymbol{Q}$ (resp. $R$ ) \} be denoted by $\operatorname{rk}_{\boldsymbol{Q}} \pi(\Lambda)$ (resp. $\operatorname{rk}_{R} \pi(\Lambda)$ ). We prove that $\mathrm{rk}_{R} \pi(\Lambda)=\mathrm{rk}_{Q} \pi(\Lambda)$, which is equivalent to the closedness of $\pi(\Lambda)$ in $\pi(\mathrm{g}) . \quad \Lambda$ is generated by $\left\{e_{1}, \cdots, e_{n}, d_{1}, \cdots, d_{r}\right\}$ 
and thus $\pi(\Lambda)$ is generated by $\left\{\tilde{e}_{1}, \cdots, \tilde{e}_{r}, \tilde{d}_{1}, \cdots, \tilde{d}_{r}\right\}$, where $\tilde{e}_{i}=\pi\left(e_{i}\right)$ etc. Thus $\mathrm{rk}_{Q} \pi(\Lambda) \leqq 2 r$. On the other hand, $\left\{\tilde{e}_{1}, \cdots, \tilde{e}_{r}, \tilde{d}_{1}, \cdots, \tilde{d}_{r}\right\}$ are linearly independent over $R$, since $\sqrt{-1}\left\{\operatorname{Im} d_{1}, \cdots, \operatorname{Im} d_{r}\right\}$ spans $\sqrt{-1} \mathfrak{a}$, and $\mathfrak{g} / \mathfrak{b}^{C} \cong \tilde{\mathfrak{a}}^{C}=\tilde{\mathfrak{a}} \oplus \sqrt{-1} \tilde{\mathfrak{a}}$, where $\tilde{\mathfrak{a}}:=\pi(\mathfrak{a}) \cong \mathfrak{a}$ is spanned by $\left\{\tilde{e}_{1}, \cdots, \tilde{e}_{r}\right\}$. Thus we have $\operatorname{rk}_{R} \pi(\Lambda)=\operatorname{rk}_{Q} \pi(\Lambda)=2 r$. Hence $\mathfrak{b}^{C}+\Lambda$ is a closed subgroup of $\mathrm{g}$ isomorphic to $\mathfrak{b}^{C} \oplus Z^{2 r}$. Let $\left(\mathfrak{b}^{C}+\Lambda\right) / \Lambda$ be denoted by $H$. Then $H$ is a Stein subgroup of $G=\mathfrak{g} / \Lambda$, and isomorphic to $C^{* n-r}$.

(ii) Now we prove that the factor group of $G$ by any maximal Stein subgroup $N$ is compact. Let $G / N$ be non-compact. Then by (i) there exists a nontrivial Stein subgroup $H$ of $G / N$. Let $\pi$ be the canonical surjection from $G$ onto $G / N$. Then $\pi^{-1}(H)$ is a Stein subgroup of $G$ containing $N$, since $\pi^{-1}(H)$ is an extension of a Stein group $H$ by a Stein group $N$ (cf. [8]). By the maximality condition of $N$, we have $\pi^{-1}(H)=N$, which is a contradiction. As for the numerical relation, we have $\delta(G / N)=\beta(G / N) \geqq \beta(G)$ by (1.4) since $\beta(N)=0$.

(iii) The existence of a Stein subgroup of codimension $\beta(G)$ is shown by making use of the construction of $H=C^{* n-r}$ in (i). Let $G$ be decomposed into the direct sum $C^{p} \oplus C^{* q} \oplus G_{e}$. Siece $G_{e}$ contains a Stein subgroup $H \cong C^{* n-r}, C^{P} \oplus C^{* q} \oplus H$ is a Stein subgroup of $G$ of codimension $r=\beta(G)=\beta\left(G_{e}\right)$.

q.e.d.

DEFinition 20. A Lie algebra $g$ is called reductive if the center of $\mathfrak{g}$ (denoted by $Z(\mathrm{~g})$ ) equals the unique maximal solvable ideal of $g$ (denoted by $\operatorname{rad}(\mathfrak{g}))$.

DEFINITION 21. A (connected) complex Lie group $G$ is called reductive if its Lie algebra $\mathrm{g}$ is reductive.

LEMMA 22 (cf. [2] p. 80). Let $\mathrm{g}$ be reductive. Then any ideal $\mathfrak{a}$ of $\mathfrak{g}$ and any quotient algebra $\mathfrak{g} / \mathfrak{a}$ are also reductive.

THEOREM 23. Let $G$ be a connected reductive complex Lie group. Then there exists a maximal normal Stein subgroup of codimension $\beta(G)$. Furthermore for any maximal normal Stein subgroup $N$ of $G$, the factor group $G / N$ is a complex torus of dimention not smaller than $\beta(G)$.

Proof. If $G$ is abelian, Theorem 23 reduces to Lemma 19 . Now we assume $G$ to be non-abelian. Denoting the Lie algebra of $G$ by $\mathfrak{g}$, we find $\operatorname{rad}(\mathfrak{g})=Z(\mathfrak{g}) \subseteq \mathfrak{g}$. So by the decomposition theorem of Levi, there exists a non-trivial semi-simple complex Lie subalgebra $\mathfrak{g}$ of $\mathfrak{g}$ such 
that $\mathfrak{g} \cap \operatorname{rad}(\mathfrak{g})=\{0\}$ and $\mathfrak{g}+\operatorname{rad}(\mathfrak{g})=\mathfrak{g}$. Since $[\mathfrak{g}, \mathfrak{g}]=[\mathfrak{g}, \mathfrak{g}+Z(\mathfrak{g})]=$ $[\mathfrak{g}, \mathfrak{g}] \subseteq \mathfrak{g}$, we find that $\mathfrak{g}$ is an ideal of $g$. Thus we conclude that $\mathfrak{g}=$ $\mathfrak{g}+Z(\mathfrak{g})$ is a direct sum decomposition of a Lie algebra $g$. By the same argument as in [7] p. 210 we prove now that the connected normal subgroup $S$ of $G$ corresponding to the ideal $\mathfrak{g}$ of $g$ is closed in $G$. Since $G$ is connected, we get $G=Z(G) S$, where $Z(G)$ denotes the connected center of $G . \quad S \cap Z(G)$ is finite, since the center of a connected complex semisimple lie group is finite. We define a group homomorphism $\rho$ from $\tilde{G}:=Z(G) \times S$ onto $G=Z(G) S$, by $\rho(x, y)=x y,(x \in Z(G), y \in S)$. Introducing the natural topology on $\tilde{G}$, we find that $\rho$ is a continuous homomorphism with the finite kernel $\cong Z(G) \cap S$. Consequently $\rho$ is a closed map. Since every semi-simple complex Lie group is Stein (cf. [8]), we obtain a nontrivial normal Stein subgroup $S$ of $G$. Now we prove that for a maximal normal Stein subgroup $N$ of $G, G / N$ is compact. For if not, $G / N$ is again reductive by Lemma 22, and hence contains a non-trivial normal Stein group $H$. Denoting the canonical surjection from $G$ onto $G / N$ by $\pi$, we find that $\pi^{-1}(H)$ is a normal Stein subgroup of $G$ (cf. [8]) which contains $N$ as a proper subgroup. This contradicts the maximality condition on $N . \quad \delta(G / N)=\beta(G / N) \leqq \beta(G)$ follows directly from (1.4) since $\beta(N)=0$. Now we construct a maximal normal Stein subgroup $L$ of condimension $\beta(G)$. Since $S \cap Z(G)$ is finite, we have $\beta(G / S)=\beta(Z(G) S / S)=\beta\left(Z(G)\right.$ ) by $(1.4)^{\prime}$. On the other hand $\beta(G) \geqq \beta(Z(G)) \geqq \beta(G e)=\beta(G)$. Thus we have $\beta(G)=\beta(G / S)$. Since $G / S$ is abelian we can apply Lemma 19 to $G / S$, to conclude that $G / S$ contains a maximal Stein subgroup $N$ such that $\delta(N)=\delta(G / S)-\beta(G)$. Denoting the canonical surjection from $G$ onto $G / S$ by $\pi$, we see that $L:=\pi^{-1}(N)$ is a normal Stein subgroup of $G$ such that $\delta(L)=\delta(N)+\delta(S)$ $=\delta(G)-\beta(G)$.

q.e.d.

EXAMPLE 24. If $\alpha(G)=0$ then $G$ is reductive as is shown implicitly in the proof of the Proposition in [7] p. 209. In this case any (maximal) normal Stein subgroup $N$ of codimension $\beta(G)$ satisfies $\alpha(N)=0$ by (1.1) $\sim(1.3)$.

CoRollary 25. Every connected complex Lie group $G$ has a structure of holomorphic fibre bundle $G=E(\pi, F, T, N)$ over a complex torus $T$ of dimension $\delta(G)-\beta(G)$, whose fibre is a Stein manifold biholomorphically isomorphic to $N \times C^{\alpha}$, where the structure group $N$ of $E$ is a 
maximal normal Stein subgroup of $K^{C}$ and $\alpha=\alpha(G) . \quad N$ acts trivially on $C^{\alpha}$, and on $N$ as a left translation.

Proof. It is an immediate consequence of Example 24 since $G$ is analytically homeomorphic to $K^{C} \times C^{\alpha}$ (cf. $\S 1$ ).

q.e.d.

Counterexample 26. If $G$ is not reductive, Theorem 23 is not true in general. Let $g$ be a three-dimensional complex solvable Lie algebra with a base $\left\{X_{1}, X_{2}, X_{3}\right\}$ such that $\left[X_{1}, X_{2}\right]=\left[X_{1}, X_{3}\right]=0$, and $\left[X_{2}, X_{3}\right]=X_{1}$. Let $\tilde{G}$ be the simply connected solvable group whose Lie algebra is $\mathrm{g}$. Then the center of $\tilde{G}=Z(\tilde{G}) \cong C$ contains a discrete subgroup $\Gamma \cong Z^{2}$ of rank 2. Let $G=\tilde{G} / \Gamma$ be the factor group of $\tilde{G}$ by $\Gamma$. Then the unique normal Stein subgroup of $G$ is trivial, but $G$ is not compact.

\section{$\S 3$. Completeness and compactness}

There are two definitions of $q$-complete complex manifolds in [1] and in [13]. We adopt here the latter one from an aesthetic point of view.

DEFINITION 27. Let $\phi$ be a $C^{\infty}$ real valued function on an $n$-dimensional complex manifold $X$ and let $\left(z_{1}, \cdots, z_{n}\right)$ be a coordinate system on some neighborhood of a point $x \in X$. $\phi$ is called strongly $q$-pseudoconvex at $x$ if the Levi form of $\phi$ :

$$
L(\phi)=\sum_{i, j=1}^{n} \frac{\partial^{2} \phi}{\partial z_{i} \partial \bar{z}_{j}} d z_{i} d \bar{z}_{j}
$$

has at least $(n-q)$ positive eigenvalues at $x$. If $\phi$ is strongly $q$ pseudoconvex at every point of $X$, we say $\phi$ is strongly $q$-pseudoconvex on $X$.

DEFINITION 28. A complex manifold $X$ is $q$-complete if there exists a strongly $q$-pseudoconvex function $\phi$ on $X$ such that for any $c \in \boldsymbol{R}$, $X(\phi, c):=\{x \in X \mid \phi(x)<c\}$ is relatively compact in $X$.

Since every complex manifold $X$ of dimension $n$ is $n$-complete (cf. [14]), we can associate to $X$ a (finite) non-negative integer $q(\leqq n)$ in the following way.

Definition 29. Completeness of $X$ (denoted by com $(X))$ is $q$, if $X$ is $q$-complete and not $(q-1)$-complete.

The following is an immediate consequence of the above definition and we shall omit the proof. 
LEMMA 30. Let $Y$ be a closed submanifold of a complex manifold $X$. Then we have $\operatorname{com}(X) \geqq \operatorname{com}(Y)$.

DeFinition 31. A complex manifold $X$ is weakly o-complete (or woc) if there exists a $C^{\infty}$-function $\phi: X \rightarrow R$ such that,

(i) $L(\phi) \geqq 0$ (i.e. positive semidefinite) on $X$

(ii) $X(\phi, c):=\{x \in X \mid \phi(x)<c\} \Subset X,{ }^{\forall} c \in R$.

DEFINITION 32. A woc manifold $X$ is $q$-compact if there exists a $C^{\infty}$-function $\phi: X \rightarrow R$ such that,

(i) $L(\phi) \geqq 0$ and $L(\phi)$ has at least $(n-q)$ positive eigen values at every point of $X$, where $n=\delta(X)$.

(ii) $X(\phi, c):=\{x \in X \mid \phi(x)<c\} \subset X, \nabla_{c} \in \boldsymbol{R}$

Definition 33. Compactness of a woc manifold $X$ (denoted by $\operatorname{comp}(X))$ is $q$, if $X$ is $q$-compact but not $(q-1)$-compact.

For a woc manifold $X$, it is evident that $\operatorname{comp}(X) \geqq \operatorname{com}(X)$ and $\operatorname{comp}(X)=0 \Longleftrightarrow \operatorname{comp}(X)=0$. The following analogy of Lemma 30 is easily verified.

LEMMA 34. Any closed submanifold of a q-compact manifold is again q-compact.

Proposition 35. Let $G$ be a connected complex Lie group. Then $\beta(G) \leqq \operatorname{com}(G)$.

Proof. Since $\operatorname{com}(G) \geqq \operatorname{com}\left(G_{e}\right)$ by Lemma 30 and $\beta(G)=\beta\left(G_{e}\right)$, it is enough to prove $\beta\left(G_{e}\right) \leqq \operatorname{com}\left(G_{e}\right)$. Let $\delta=\delta\left(G_{e}\right)$ and $\gamma=\gamma\left(G_{e}\right)$. Then $G_{e}$ has the same homotopy type as a $\gamma$-dimensional real torus, hence $H^{r}\left(G_{e}, C\right)=C$. On the other hand, $H^{\delta+q}\left(G_{e}, C\right)=0$ if $q>\operatorname{com}\left(G_{e}\right)$ (cf. [13]). Thus we obtain that $\gamma \leqq \delta+\operatorname{com}\left(G_{e}\right)$. Since $\alpha\left(G_{e}\right)=0$, $\beta\left(G_{e}\right)=\gamma\left(G_{e}\right)-\delta\left(G_{e}\right)=\gamma-\delta \leqq \operatorname{com}\left(G_{e}\right)$ by (1.1). q.e.d.

THEOREM 36. Let $G$ be a connected complex Lie group, and let $P=P(B, G)$ be a holomorphic principal bundle over a complex manifold $B$ with the structure group $G$. Then we have

$$
\operatorname{com}(P) \leqq \operatorname{com}(B)+\beta(G) \text {. }
$$

Proof. (Step 1) First we assume the assertion of the theorem for simple structure groups of arbitrary dimensions (a simple group means a Lie group which contains no non-trivial connected normal subgroup). 
We prove the assertion for non-simple structure groups by the induction on $\delta(G)$. If $\delta(G)=0$ there is nothing to prove. Let us assume Theorem 36 for any $G$ such that $\delta(G) \leqq n$. If $\delta(G)=n+1$ and $G$ is neither Stein nor $(H, C)$ then $1 \leqq \delta\left(G_{e}\right) \leqq n$ and we get

$$
\begin{aligned}
& \operatorname{com}\left(P / G_{e}\right) \leqq \operatorname{com}(B)+\beta\left(G / G_{e}\right) \\
& \operatorname{com}(P) \leqq \operatorname{com}\left(P / G_{e}\right)+\beta\left(G_{e}\right)
\end{aligned}
$$

from the induction hypothesis. Here we denote by $P / G_{e}$ the quotient holomorphic principal bundle $P(B, G) / G_{e}:=P / G_{e}\left(B, G / G_{e}\right)$ of $P=P(B, G)$, induced naturally by the normal subgroup $G_{e}$. $P$ can be considered in a natural fashion as a holomorphic principal bundle over $P / G_{e}$, with the structure group $G_{e}$. Combining (3.2) and (3.3) we get (3.1) since $\beta(G)$ $=\beta\left(G_{e}\right), \beta\left(G / G_{e}\right)=0$. If $G$ is Stein but not simple, there exists a closed normal connected complex Lie subgroup $A$ of $G$ such that (i) $\{e\} \subseteq A \sqsubseteq G$, (ii) $G / A$ is Stein (cf. [8], Proposition 7). By the induction hypothesis we obtain similarly as before

$$
\begin{aligned}
& \operatorname{com}(P / A) \leqq \operatorname{com}(B)+\beta(G / A) \\
& \operatorname{com}(P) \leqq \operatorname{com}(P / A)+\beta(A) .
\end{aligned}
$$

Thus we get (3.1) since $\beta(A)=\beta(G)=\beta(G / A)=0$. If $G$ is $(H, C)$ and not compact, there exists a maximal Stein subgroup $N$ of $G$ such that $\delta(G / N)=\beta(G / N)=\beta(G)$. By the induction hypothesis we obtain

$$
\begin{aligned}
& \operatorname{com}(P / N) \leqq \operatorname{com}(B)+\beta(G / N) \\
& \operatorname{com}(P) \leqq \operatorname{com}(P / N)+\beta(N) .
\end{aligned}
$$

Since $\beta(N)=0$, we obtain (3.1) combining the above inequalities. Let us assume now $G$ to be compact. If $B$ is $q$-complete with respect to a strongly $q$-pseudoconvex function $\phi: B \rightarrow R$, such that $B(\phi, c):=$ $\{x \in B \mid \phi(x)<c\} \subset B$, for $\forall c \in R$, denoting the projection mapping from $P$ onto $B$ by $\pi$, we can induce a strongly $(q+\delta(G))$-pseudoconvex function $\pi^{*} \phi=\phi \circ \pi: P \rightarrow R . \quad P\left(\pi^{*} \phi, c\right):=\left\{p \in P \mid \pi^{*} \phi(p)<c\right\}=\pi^{-1}(B(\phi, c)) \subset P$, since $\pi$ is a proper mapping. Thus $\operatorname{com}(P) \leqq \operatorname{com}(B)+\delta(G)$, which implies (3.1) since $\delta(G)=\beta(G)$. Thus Step 1 is complete.

Before proceding to Step 2 we introduce the following notion.

Definition 37. We say that a $C^{\infty}$-function $\phi: P(B, G) \rightarrow \boldsymbol{R}$ is strongly $q$-pseudoconvex along the fibre if the restriction of $\phi$ to each 
fibre of $P(B, G)$ is strongly $q$-pseudoconvex.

Now we consider $P=P(B, G)$, where $G=G L(n, C)$. Since $B$ is assumed to be paracompact, we can choose a locally finite covering $\left\{U_{\alpha}\right\}_{\alpha \in I}$ of $B$ in such a way that $P$ is trivial on each $U_{\alpha}$. We can take a positive difinite $C^{\infty}$-hermitian metric along the fibre of $P$; in terms of the covering $\left\{U_{\alpha}\right\}_{\alpha \in I}$ it is represented as the system of $C^{\infty}$-mappings $\left\{h_{\alpha}\right\}_{\alpha \in I}$ from each $U_{\alpha}$ to the space of positive definite hermitian matrices such that $h_{\beta}={ }^{t} \bar{g}_{\alpha \beta} h_{\alpha} g_{\alpha \beta}$, if $P$ is represented by the holomorphic cocycle $g_{\alpha \beta}: U_{\alpha} \cap U_{\beta} \rightarrow G L(n, C)$. Let $\phi_{\alpha}$ be the identification mapping from $U_{\alpha} \times G$ onto $\bar{\pi}^{1}\left(U_{\alpha}\right)$. For $x \in U_{\alpha} \cap U_{\beta}, p=\phi_{\alpha}\left(x, \xi^{(\alpha)}\right)=\phi_{\beta}\left(x, \xi^{(\beta)}\right)$ for some $\xi^{(\alpha)}$, $\xi^{(\beta)} \in G=G L(n, C)$, that satisfy $\xi^{(\alpha)}=g_{\alpha \beta}(x) \xi^{(\beta)}$. Since ${ }^{t} \bar{\xi}^{(\alpha)} h_{\alpha}(x) \xi^{(\alpha)}=$ ${ }^{t} \bar{\xi}^{(\beta)} h_{\beta}(x) \xi^{(\beta)}$, we can define a global $C^{\infty}$ function $\Phi_{h}: P \rightarrow R$ by setting $\Phi_{h}(p)=\operatorname{trace}^{t \xi^{(\alpha)}} h_{\alpha}(x) \xi^{(\alpha)}$ for $p=\phi_{\alpha}\left(x, \xi^{(\alpha)}\right)$. Under this situation we prove the following

LEMMA 38. $\Phi_{h}$ is a strogly 0-pseudoconvex function along the fibre of $P=P(B, G)$ and consequently strongly $m$-pseudoconvex on $P$, where $m=\delta(B)$.

Proof. We can assume, without loss of generality, each $U_{\alpha}$ to be a coordinate neighborhood. We fix a local coordinate system $\left(z_{1}, \cdots, z_{m}\right.$, $\left.z_{m+1}, \cdots, z_{m+n 2}\right)$ on the neighbourhood $\pi^{-1}\left(U_{\alpha}\right)$ of $p \in P$, by taking $\left(z_{1}, \cdots, z_{m}\right)$ as the coordinate system on $U_{\alpha}$, and $z_{m+1}:=\xi_{11}^{(\alpha)}, z_{m+2}:=\xi_{21}^{(\alpha)}, \cdots, z_{m+n}$ : $=\xi_{n 1}^{(\alpha)}, z_{m+n+1}:=\xi_{12}^{(\alpha)}, \cdots, z_{m+2 n}:=\xi_{n 2}^{(\alpha)}, \cdots, z_{m+n(n-1)+1}:=\xi_{1 n}^{(\alpha)}, \cdots, z_{m+n 2}:=\xi_{n n}^{(\alpha)}$, where $\xi_{i j}^{(\alpha)}$ denotes the $(i, j)$-component of the matrix $\xi^{(\alpha)} \in G L(n, C)$. Then representing the Levi form of $\Phi_{h}$ in terms of this coordinate system, we get

$$
L\left(\Phi_{h}\right)=\left(\begin{array}{c:cccc}
A & & & D \\
& & { }^{t} h_{\alpha} & & \\
& & \ddots & & \\
& & \ddots & \\
{ }^{t} \bar{D} & & & \ddots & \\
& 0 & & & { }^{t} h_{\alpha}
\end{array}\right)
$$

where $A$ and $D$ denote some matrices of type $(m, m)$ and $\left(n^{2}, m\right)$ respectively. Since $h_{\alpha}$ is positive definite, $L\left(\Phi_{h}\right)$ is positive definite if restictited to the holomorphic tangent space of the fibre of $P$, which means $\Phi_{h}$ is strongly 0-pseudoconvex along the fibre. Consequently $\Phi_{h}$ is strongly 
$m$-pseudoconvex on the total space $P$.

Now we proceed to

Proof of Theorem 36 (Step 2). There remains only to prove the theorem for simple structure groups. It is well known that every connected complex semisimple Lie group is (isomorphic to) a closed complex subgroup of $G L(n, C)$ for some $n$. On the other hand simple groups that are not semisimple are one-dimensional i.e. $C, C^{*}$ and complex tori. For a compact structure group $G$, the assertion of the theorem is already verified, while $C$ and $C^{*}$ are isomorphic to closed subgroups of $G L(n, C)$ for some $n$. So it is sufficient to prove the theorem for such $P(B, G)$ as $G$ is a closed (connected) complex subgroup of $G L(n, C)$ for arbitrary $n$. Now assume the theorem for $P_{1}(B, G L(n, C))$. If $G$ is a closed complex subgroup of $G L(n, C)$, then $P:=P(B, G)$ can be considered as a closed complex submanifold of $P_{1}=P_{1}(B, G L(n, C))$ whose representing holomorphic cocycle is the same as that of $P(B, G)$. Thus we have $\operatorname{com}(P) \leqq \operatorname{com}\left(P_{1}\right) \leqq \operatorname{com}(B)$ (cf. Lemma 30 ), since $G L(n, C)$ is Stein. Now we must prove the theorem for $P_{1}(B, G L(n, C))$. We define a group homomorphism $\omega$ from $G L(n, C)$ into $S L(n+1, C)$ by setting $\omega(g)=\left(\begin{array}{ll}g & 0 \\ 0 & \operatorname{det} g\end{array}-1\right)$ for $g \in G L(n, C)$. Since the image of $\omega$ is closed in $S L(n+1, C)$ and $\omega$ is injective, we can consider $P_{1}(B, S L(n+1, C))$ as a closed submanifold of $P_{2}:=P_{2}(B, S L(n+1, C))$ and consequently of $P_{3}:=P_{3}(B, G L(n+1, C))$ which are trivial extensions of $P$ in the sense that their representing holomorphic cocycles are the same as that of $P$. By restricting the strongly $m$-pseudoconvex function $\Phi_{h}$ on $P_{3}$, defined as above, to $P_{1}$, we get a strongly $m$-pseudoconvex function $\Phi_{h} \mid P_{1}$ (denoted by the same symbol if there is no fear of confusion), on $P_{1}$, where $m=\delta(B)$. By the remark in [1] p. 257 we can choose a coordinate of the fibre $\pi^{-1}(x)$ in such a way that $\left(\partial h_{\alpha}\right)_{x}=0\left(x \in U_{\alpha}\right)$, which implies $D=0$ for $D$ in (3.4). Thus we obtain

$$
L\left(\Phi_{h}\right)=\left(\begin{array}{c:ccc}
A & & & 0 \\
& { }^{t} h_{\alpha} & & \\
& & \ddots & \\
0 & 0 & & \\
& & & \\
& & & \\
& & & \\
& & &
\end{array}\right)
$$

with respect to such a coordinate system. We can consider $A$ as the 
hermitian form on the holomorphic tangent space $T_{x}(B)$ of $B$ at $x$. Let $B$ be $q$-complete with respect to a strongly $q$-pseudoconvex function $\phi$. Then we can construct a $C^{\infty}$-function $\psi: R \rightarrow R$ such that, if $u=\psi \circ \phi$, $L(u)+A$ has $(m-q)$ positive eigenvalues and $B(u, c)=\{x \in B ; u(x)<c\}$ $\subset B$ (cf. [15]). We can assume $u>0$ without loss of generality. Let $\Psi$ be the $C^{\infty}$ real valued function on $P_{1}$, defined by $\Psi:=u \circ \pi+\Phi_{h}$. Then the Levi form of $\Psi$ :

$$
L(\Psi)=\left(\begin{array}{c:c:ccc}
L(u)+A & & 0 & \\
\hdashline 0 & & \ddots & \\
& & 0 & & { }^{t} h_{\alpha}
\end{array}\right)
$$

has $\left(n^{2}+m-q\right)$ positive eigenvalues, which implies $\Psi$ is strongly $q$ pseudoconvex on $P_{1}$. On the other hand $P_{1}(\Psi, c):=\left\{p \in P_{1} ; \Psi(p)<c\right\}$ is relatively compact in $P_{1}$, since $P_{1}(\Psi, c) \subset P_{1}(u \circ \pi, c) \cap P_{1}\left(\Phi_{h}, c\right), B(u, c) \subset B$, $P_{1}\left(\Phi_{h}, c\right) \subset P_{2}\left(\Phi_{h}, c\right) \cap P_{1}$, and $\pi^{-1}(x)\left(\Phi_{h}, c\right):=\left\{p \in \pi^{-1}(x) \cong S L(n+1, C)\right.$; $\left.\Phi_{h}(p)<c\right\} \Subset \pi^{-1}(x) \cong S L(n+1, C)$, where $\pi$ denotes the projection from $P_{2}=P_{2}(B, S L(n+1, C))$ onto $B$. Thus we have $\operatorname{com}\left(P_{1}\right) \leqq \operatorname{com}(B)$. Step 2 is now complete, and the theorem is proved.

CoRollary 39. $\beta(G)=\operatorname{com}(G)$, and hence $\beta$ is a complex analytic invariant of $G$, i.e. if $G \approx G^{\prime}$ (as complex manifolds), then $\beta(G)=\beta\left(G^{\prime}\right)$ (cf. Proposition 17).

Proof. By Proposition 35 we have already $\beta(G) \leqq \operatorname{com}(G)$, while the converse inequality is obtained as a trivial case of the theorem.

q.e.d.

COROLlary 40. $\alpha$ is a complex analytic invariant of $G$.

Proof. As $\alpha=\beta+\delta-\gamma$ by (1.1), the invariance of $\alpha$ follows from the above Corollary and Proposition 13.

q.e.d.

Corollary 14. If $B$ is Stein, $\operatorname{com}(P)=\beta(G)$.

Proof. Since $G$ is a fibre of $P, \operatorname{com}(G) \leqq \operatorname{com}(P)$ by Lemma 30 . On the other hand $\operatorname{com}(P) \leqq \beta(G)$, since $\operatorname{com}(B)=0$. $\quad$ q.e.d.

We shall use later the following which is easily verified.

LEMMA 42. Let $M$ and $N$ be complex manifolds. Then com $(M \times N)$ $\leqq \operatorname{com}(M)+\operatorname{com}(N)$. If both $M$ and $N$ are woc, so is $M \times N$ and we 
have comp $(M \times N) \leqq \operatorname{comp}(M)+\operatorname{comp}(N)$.

LEMMA 43. Let $\tilde{X}$ and $X$ be complex manifolds such that $(\tilde{X}, \pi, X)$ is a finite holomorphic covering. Then $\tilde{X}$ is woc $\Longleftrightarrow X$ is woc. Furthermore if $\tilde{X}($ or $X)$ is woc, then $\operatorname{comp}(\tilde{X})=\operatorname{comp}(X)$.

Proof. If $X$ is woc with respect to a pseudoconvex function $\phi$, then $\pi^{*} \phi=\phi \circ \pi$ is pseudoconvex (i.e. $L(\phi \circ \pi) \geqq 0$ ) and $L(\phi)=L(\phi \circ \pi)$, if we identify the tangent space of $\tilde{X}$ at $p \in \tilde{X}$, with that of $X$ at $\pi(p) \in X$. Relative compactness of $\tilde{X}(\phi \circ \pi, c):=\{p \in \tilde{X} \mid \phi \circ \pi(p)<c\}$ in $\tilde{X}$ is evident since $\pi$ is a proper mapping from $\tilde{X}$ onto $X$. Thus $\tilde{X}$ is woc with respect to $\pi^{*} \phi$ and we obtain $\operatorname{comp}(\tilde{X}) \leqq \operatorname{comp}(X)$. On the other hand, let $\tilde{X}$ be woc with respect to a pseudoconvex function $\phi$. Then we can define a $C^{\infty}$ function $\tilde{\phi}: X \rightarrow R$, by setting

$$
\tilde{\phi}(x)=\sum_{p_{i} \in \pi^{-1}(x)} \phi\left(p_{i}\right) \quad \text { for } x \in X
$$

We have $L(\tilde{\phi}, x)=\sum_{p_{i} \in \pi^{-1}(x)} L\left(\phi, p_{i}\right)$, if we identify the tangent space of $X$ at $x$ with those of $\tilde{X}$ at $p_{i} \in \pi^{-1}(x)$. Since every $L\left(\phi, p_{i}\right)\left(p_{i} \in \pi^{-1}(x)\right)$ is positive semidefinite, so is $L(\tilde{\phi}, x)$. If every $L\left(\phi, p_{i}\right)\left(p_{i} \in \pi^{-1}(x)\right)$ has $q$ positive eigen values, so does $L(\tilde{\phi}, x)$. Relative compactness of $X(\tilde{\phi}, c)$ : $=\{x \in X \mid \tilde{\phi}(x)<c\}$ in $X$ is evident. Thus $X$ is woc with respect to the exhausting function $\tilde{\phi}$ and we have $\operatorname{comp}(\tilde{X}) \geqq \operatorname{comp}(X)$. q.e.d.

The following is due to Kazama [4].

LEMMA 44. Every connected complex abelian Lie group $G$ is woc and $\operatorname{com}(G) \leqq \beta(G)$.

THEOREM 45 (cf. [5]). Every connected complex Lie group $G$ is woc and $\operatorname{comp}(G)=\operatorname{com}(G)=\beta(G)$.

Proof. As $G$ is analytically homeomorphic to $K^{c} \times C^{\alpha}$, we prove first that $K^{C}$ is woc, and secondly that comp $\left(K^{c}\right)=\beta(G)$. Since $K^{C}$ is reductive, by the same argument as in the proof of Theorem 23 we have $K^{C}=Z S$, where $Z$ is the connected center of $K^{c}$ and $S$ is the maximal semisimple normal connected subgroup of $K^{c}$. Letting $\rho$ be the analytic homomorphism from $Z \times S$ onto $K^{C}=Z S$, we have $\operatorname{comp}(Z \times S)=$ $\operatorname{comp}\left(K^{C}\right)$, since $\operatorname{ker} \rho$ is finite and $Z \times S$ is woc (cf. Lemma 42,43 and 44). On the other hand $\operatorname{comp}(Z \times S)=\operatorname{comp}(Z) \leqq \beta(Z) \leqq \beta(G)=\operatorname{com}(G)$, and $\operatorname{comp}(G) \leqq \operatorname{comp}\left(K^{C}\right)+\operatorname{comp}\left(C^{\alpha}\right)=\operatorname{comp}\left(K^{c}\right)$. Thus we obtain 
$\operatorname{comp}(G) \leqq \beta(G)=\operatorname{com}(G) \leqq \operatorname{comp}(G)$.

q.e.d.

REMARK. In [5] Kazama proved that every $G$ is woc and that comp $(G) \leqq \tau(G)$, independently of his original result (i.e. Lemma 44 ).

\section{§4. Some extension and problems}

There remains the problem whether Theorem 36 is true, without the assumption of connectedness on $G$. By the same argument as in the proof of Lemma 43 we can prove easily

LemMa 46. Let $\tilde{X}$ and $X$ be complex manifolds, such that $(\tilde{X}, \pi, X)$ is a finite holomorphic covering. Then we have $\operatorname{com}(\tilde{X}) \leqq \operatorname{com}(X)$.

We can generalize Theorem 36 in the following way.

THEOREM 47. Let $P=P(B, G)$ be a holomorphic principal bundle such that $G / G^{0}$ is a discrete subgroup of $G L(n, C)$ for some $n>0$. Then $\operatorname{com}(P) \leqq \operatorname{com}(B)+\beta(G)$.

Proof. Let $P / G^{0}=P / G^{0}\left(B, G / G^{0}\right)$ be the quotient bundle of $P$ induced by $G^{0}$. Then $P$ is a principal bundle over $P / G^{0}$ with the structure group $G^{0}$. We can apply Theorem 36 to $P=P\left(P / G^{0}, G^{0}\right)$, since $G^{0}$ is connected. Hence we obtain $\operatorname{com}(P) \leqq \operatorname{com}\left(P / G^{0}\right)+\beta\left(G^{0}\right)$. On the other hand $G / G^{0}$ is a discrete subgroup of $G L(n, C)$ and hence we can consider $P / G^{0}$ as the closed submanifold of the holomorphic principal bundle $P^{\prime}:=P^{\prime}(B, G L(n, C))$ which is a trivial extension of $P / G^{0}$ (cf. § 3). Thus we obtain $\operatorname{com}(P) \leqq \operatorname{com}(B)+\beta(G)$, since $\beta(G)=\beta\left(G^{0}\right) \quad$ q.e.d.

Now we may ask whether $\operatorname{com}(P)=\operatorname{com}(B)+\beta(G)$ is true or not. This is not true in general. For example $P:=C^{* 2}$, considered as a complex Lie group, has a closed (normal) subgroup $G \cong C$ and the factor space $B:=C^{* 2} / C$ is a one-dimensional complex torus, hence $\operatorname{com}(P)=$ $0<\operatorname{com}(B)=1$. By [8], if $\alpha(G)=\operatorname{com}(P)=0$, we have $\operatorname{com}(B)=0$. Can we generalize this result in the following way; if $\alpha(G)=0$ then $\operatorname{com}(P)=\operatorname{com}(B)+\beta(G)$ ? This conjecture is based also on the fact that $\alpha(N)=0$ induces $\beta(N, G)=0$ for a connected complex Lie group $G$ and its closed connected normal complex Lie subgroup $N$ (cf. Prop. 9).

The last problem is to generalize Theorem 36 (or 47) to arbitrary holomorphic fibre bundles $E=E(\pi, F, B, G)$ over a base space $B$ with fibre $F$ and structure group $G$. We may conjecture that $\operatorname{com}(E) \leqq \operatorname{com}(B)$ $+\operatorname{com}\left(F^{\prime}\right)$. This conjecture was solved affirmatively by Villani [15] for 
holomorphic vector bundles. The above inequality is a generalization of the well known result of Matsushima and Morimoto [8] which asserts $\operatorname{com}(E)=\operatorname{com}(B)+\operatorname{com}(F)$ in case $B$ and $F$ are Stein, and $G$ is connected.

\section{REFERENCES}

[1] Andreotti, A., and Grauert, H., "Théorèmes de finitude pour la cohomologie des espaces complexes," Bull. Soc. Math. France 90 (1962), 139-259.

[ 2 ] Bourbaki, N., Groupes et algebres de Lie (Chap. 1), Hermann, Paris, 1961.

[ 3 ] Iwasawa, K., "On some types of topological groups," Ann. Math. 50 (1949), 507558.

[4] Kazama, H., "On pseudo convexity of complex abelian Lie groups," J. Math. Soc. Japan 25 (1973), 329-333.

[ 5 ] — "On pseudoconvexity of complex Lie groups, "Mem. Fac. Sci. Kyushu Univ. 27 (1973), 241-247.

[6] Kopfermann, K., "Maximale Untergruppen Abelscher komplexer Liescher Gruppen," Schr. Math. Inst. Univ. Münster 39 (1964).

[7] Matsushima, Y., "Espaces homogènes de Stein des groupes de Lie complexes," Nagoya Math. J. 16 (1960), 205-218.

[8] - and Morimoto, A., "Sur certains espaces fibrés holomorphes sur une variété de Stein," Bull. Soc. Math. France 88 (1960), 137-155.

[ 9 ] Morimoto, A., “On various complex Lie groups," Sugaku 15 (1963-1964), 202-214. (in Japanese)

[10] _-, "Non-compact complex Lie groups without non-constant holomorphic functions," Proc. Conf. Complex Analysis at Univ. Minn., Springer, Berlin, 1965; pp. 257-272.

[11] Narasimhan, R., "The Levi problems for complex spaces II," Math. Ann. 146 (1962), 195-216.

[12] Rosenlicht, M., "Some basic theorems on algebraic groups," Amer. J. Math. 78 (1956), 401-443.

[13] Vesentini, E., On Levi convexity of complex manifolds and cohomology vanishing theorem. Tata Institute Lecture Notes, 1967.

[14] Villani, V., "Su alcune proprietà coomologiche dei fasci coerenti su uno spazio complesso," Rend. Sem. Mat. Universita di Padova 35 (1964), 47-55.

[15] - "Fibrati vettoriali olomorfi su una varietà complessa q-completa," Ann. Scoula Norm. Sup. Pisa S. 3 vol. 20 (1966), 15-23.

Gifu University 